\title{
Non-surgical and Non-pharmacological Treatment of Knee Pain
}

\section{David Golding and Paul YF Lee*}

Welshbone, South Wales Orthopaedics Research Network, Wales, UK

"Corresponding author: Paul YF Lee, Welshbone, South Wales Orthopaedics Research Network, Wales, UK, Tel: +447764614688; E-mail: paul@welshbone.com

Received date: September 27, 2016; Accepted date: November 19, 2016; Published date: November 25, 2016

Copyright: @ 2016 Golding D, et al. This is an open-access article distributed under the terms of the Creative Commons Attribution License, which permits unrestricted use, distribution, and reproduction in any medium, provided the original author and source are credited.

\begin{abstract}
Knee pain is an increasingly common presentation to general practitioners both in the United Kingdom and worldwide and is thought to be caused by a combination of the obesity epidemic, the ageing population and continuing worldwide population growth. Two distinct aetiologies of knee pain have been described: a bony-type which is usually degenerative in nature; and a ligamentous-type which usually occurs as a result of acute injury but then predisposes to the development of bony-type in later life. There are a wide variety of treatment options available to the clinician: ranging from conservative to major surgery. Total Knee Replacement (TKR) is often the end-point of many causes of knee pain and is used with increasingly frequency. However, there are a wide variety of problems associated with TKR including ongoing pain, patient dissatisfaction and the need for revision surgery. This review aims to demonstrate that TKR should be avoided unless absolutely necessary and also provides the clinician with an overview of the various evidence-based conservative options available to be utilised to improve patient pain and functional knee capabilities.
\end{abstract}

Keywords: Knee; Non-surgical; Non-pharmacological; Treatment; Pain; Brace; Taping; Insoles; Electrical therapy

\section{Introduction}

Knee pain is one of the most common musculoskeletal presentations to general practitioners in the United Kingdom, with one study showing that $47 \%$ of over 50 -year-olds experienced knee pain within the previous year and a third of those sought medical help from their General Practitioners (GP) [1]. This represents a large proportion of GP workload and as such the recognition of the aetiology and subsequent provision of optimal management is crucial to providing an effective healthcare service.

Knee pain can be divided into two broad categories: a bony type which is typically osteoarthritis; and a soft tissue type which is predominantly ligamentous in origin. Osteoarthritis is the most common cause of knee pain in the older adult [2] with Arthritis Research UK predicting the prevalence of OA of the knee will increase from 4.7 million in 2010 to 6.5 million in 2020 due to a combination of the obesity epidemic, an ageing population and continuing population growth [3].

Soft tissue injuries most frequently occur as a result of trauma and accidents frequently involving sporting activity or exercise. Peat et al. found that the peak of soft tissue injuries occurs in the 15-19 age group for both males and females, which they reasoned coincided with the highest rates of participation in sport within these age groups [4]. Soft tissue knee injuries while younger are also associated with an almost 3 fold increased risk of developing OA during later life [5].

\section{Problem of Knee Replacements}

Severe osteoarthritis can lead to marked pain, deformity and a loss of function of the knee joint. This is turn can have a detrimental effect on mobility and overall quality of life. The eventual treatment for severe $\mathrm{OA}$ of the knee is frequently total knee replacement (TKR).
Data from the National Joint Registry (NJR) for England, Wales and Northern Ireland found that 103,126 knee replacements were carried out during 2014 with the indication for primary surgery being knee $\mathrm{OA}$ in $98 \%$ of cases [6]. Despite the high frequency that these operations were carried out, Williams et al. found that around $20 \%$ of patients were dissatisfied with their post-surgical outcomes.

In the under 55 age group, over a fifth of patients were left dissatisfied, indicating that current practice and techniques may not be as appropriate for younger more active patients groups [7]. It is well known that younger age of primary TKR is associated with a far greater incidence of the need for revision surgery. Within England, Wales and Northern Ireland the rate of revision for the under 55 age group is six times the rate compared to the over 75 group at 10 years post-operatively [8], which Khan et al. postulate is due to the greater expectations regarding a return to activity following surgery [9].

The need for revision may lead to an increasing level of dissatisfaction with the implant and a reduction in quality of life and mobility. Between $8-34 \%$ of patients experience chronic pain or disability follows TKR even with adequate radiographic and clinical evaluations and no evidence of infection $[10,11]$, indicating that surgeons are currently delivering sub-optimal results for their patients. Studies have also shown that many patients experience disappointment due to unrealistic expectations of their abilities and/or recovery postTKR surgery [12] indicating a lack of sufficient pre-operative patient education and counselling by the surgical team.

From this data it is evident that TKR should be delayed for a long as reasonably possible in younger patients with $\mathrm{OA}$ or soft tissue cartilage injuries. Fortunately there are a wide range of conservative nonsurgical options available to the clinician which this article will aim to cover. Other reviews have already covered the breadth of conventional pharmacological options available so will not be reviewed here. 


\section{Management Options}

\section{Weight loss}

The climbing obesity epidemic is undoubtedly one of the main factors leading to an increasing prevalence of knee OA. Biomechanical principles tell us that the greater the load acting on a joint, the greater the compressive forces within the joint. This leads to a greater amount of degenerative damage and subsequent development of OA. Fortunately several studies have shown that this is a reversible process and that each unit of weight loss leads to between 2-4 units of force reduction acting within the knee joint $[13,14]$. However, more recent work by DeVita et al. found that behavioral adaptations following weight loss lead to increased stride length and stride velocity and that as a result the reduction in knee forces was attenuated to a 1:1 ratio [15]. This is still significant and clinicians should encourage overweight patients with knee OA to lose weight regardless for many other health benefits such as a reduction in cardiovascular disease and diabetes prevalence. Atukorala et al. also demonstrated a dosedependence between weight loss and a reduction in knee pain and symptoms during an 18 week programme, indicating this is an important and valid lifestyle intervention to treat knee pain [16].

\section{Physiotherapy}

Physiotherapy of the knee aims to re-balance the muscle strength in the upper leg which is often dominated by the quadriceps. These methods form the core of the current management to reduce knee pain in both OA and in ligamentous injuries, utilising different methods to achieve the same overall goal. Knoop et al. found that the increase in quadriceps and hamstring strength was associated with a reduction in pain, improvements in the Western Ontario and McMaster Universities Osteoarthritis Index (WOMAC) and improved performance in the get-up-and-go test [17]. Physiotherapy is also widely used in the conservative management of ligamentous knee injuries to increase muscle strength, improve proprioception and reduce swelling: ultimately aiming for a full return to physical activity. Smith et al. systematically reviewed operative versus non-operative treatment for ACL injuries and found no difference in functional outcomes [18], indicating that surgery should only be performed when clinical instability and limitations are present.

\section{Taping}

Another intervention that has gained a foothold in conservative management of knee pain is taping, of which there are various methods. Some of these methods aim to support the ligaments of the knee or to unload the patellar in instances of patellofemoral instability [19] and are frequently applied by athletes before training or participating with a mild soft tissue injury to good effect. However, the potential beneficial use of kinesiological taping methods for adults with osteoarthritis has seen divided opinion within the literature. Kinesiology taping has been shown to lead to short term improvements in pain, walking ability and range-of-motion (ROM) in adults with knee OA [20,21] and Cho et al. add that knee joint proprioception is also improved [22]. In contrast other similar studies found no beneficial effects in kinesiology taping in pain reduction or functional status compared to sham taping $[23,24]$. However, both groups noticed that sham taping led to improved outcomes in all groups. This may indicate that kinesiology taping does not specifically improve outcomes and that all taping regimes could hold promise in the treatment of knee OA; or that there is a psychological benefit from gained from taping that leads to subjective improvements. It is very difficult to standardise taping methodologies across studies however this field could clearly benefit from a systematic review examining the differences in opinion.

\section{Braces}

Knee braces are a simple but effective adjunct in the treatment of knee pain. The simplest designs involve a single sleeve made of neoprene that encases the knee joint: these are readily available over the counter. There are a variety of benefits for the use of these type of braces such as reduced pain and postural sway [25], and while this study demonstrated no increase in joint proprioception Herrington et al. found a significant proprioceptive benefit as a result of brace wearing [26], albeit their study measured proprioception in healthy participants without knee pathology. These changes in pain and proprioception are thought to be mediated by activation of type A delta sensory fibres from skin mechanoreceptors [27].

More complex brace designs are focused on supporting ligamentous laxity and include rigid bars and straps as a protective mechanism. These braces are designed specifically to protect a certain ligament therefore it is the vital the correct diagnosis is made before the prescription of the knee brace. Misdiagnosis and incorrect prescription could lead to an exacerbation of the original injury if the ligament is not protected adequately. ACL braces are the most commonly prescribed and Pierrat et al. found that these designs are able to resist anterior drawer in an equal fashion to a native ACL at low forces, making them suitable for individuals rehabilitating from ACL injuries or sprains [28]. They also found by testing several braces that the most effective brace varied between individuals, highlighting the need for personalised fitting and testing regimes. These braces can also be worn during higher intensity physical activity and Hanzlikova et al. found that they significantly reduced knee valgus and tibial internal rotation when compared to not wearing a brace [29]. However Smith et al. concluded that current ACL brace designs do not sufficiently improve long term outcomes and rates of re-injury and state more research is needed into optimum brace design [30].

Braces are also available for PCL injuries however Jansson et al. found that none currently available offer the optimum biomechanical properties required to mimic the native PCL [31]. They also found despite the recommendation of the use of PCL braces by physicians the evidence base is slim in respect to their effectiveness. There are several PCL braces designed to be used in return to sporting activities which apply anterior force to the posterior tibia and a posterior force to the distal femur, thus aiming to protect the healing ligament; though these have not been effectively validated from either a biomechanical or clinical perspective. These braces also do not confer any protection against the subsequently common development of knee OA after PCL rupture [32]. Despite these apparent shortcomings several studies have demonstrated the benefits of PCL bracing leading to improvements in knee functional capabilities and a reduced posterior sag following bracing for four months [33]. The recent generation of PCL Brace (Figure 1) has a more robust control on the anterior and posterior translation of the tibia and therefore can give better biomechanics controls. Braces to support the other ligamentous structures in the knee such as the MCL and LCL are also available through similarly are not as well researched as ACL braces (Figure 1). 


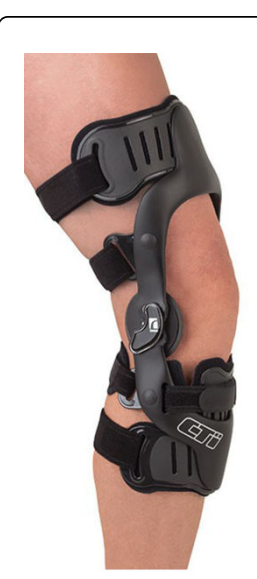

ACL Brace

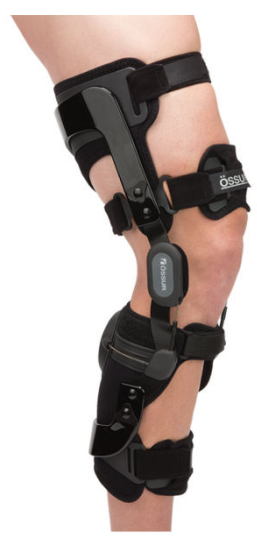

PCL Brace

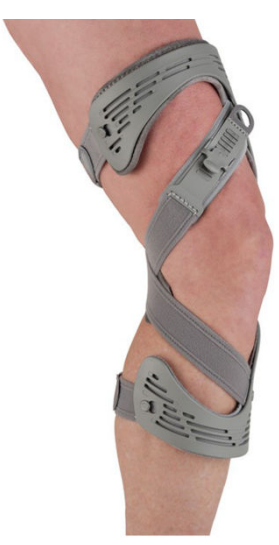

Unloader Brace
Figure 1: Different types of medical grade knee brace.

Different kinds of braces have also been designed specifically for the relief of pain in knee OA (Figure 1). The unloader type of knee brace is designed to treat unicompartmental knee $\mathrm{OA}$ by reducing the amount of stress and force passing through the affected compartment. Valgus braces are used to treatment medial compartment OA but directing forces away from the medial side by reducing the varus/adduction moment passing through the knee [34]. This leads to a widening of the joint space on the medial compartment and a subsequent reduction in the pain presenting from medial compartment OA. Raja and Dewan reviewed the clinical outcomes as a result of knee bracing and found the majority of studies found significant reductions in pain while walking and using stairs when compared to no bracing or the use of a neoprene sleeve alone [35]. The study by Ramsey et al. also added that valgus bracing reduces co-contractions of vastus medialis and vastus lateralis, and may be an additional method for reducing pain felt by brace wearers [36]. Many studies have also shown vast improvements in the functional status of the knee such a muscle strength, proprioception and walking speed [37]. However despite the well documented benefits of valgus bracing, patient compliance is still remarkable low with Squyer et al. finding that only $28 \%$ of patients used the brace regularly [38] (defined as twice per week for $>1$ hour). This is a remarkably low compliance considering braces are designed to be worn far more regularly than the frequency stated. The authors found common reasons for lack of compliance included lack of symptomatic relief, poor fit, skin irritation and discomfort. This indicates the need for a personalised approach to brace prescription with Draganich et al. finding that while standard off-the-shelf braces provided a significant amount of benefit to the patient, custom-fit braces were superior in terms of pain reduction and functional status [39].

Another type of knee brace designed to treat patellofemoral pain is the reactive knee brace. These braces use an elastomeric web to stabilise the knee and act as a shock absorber [40]. Force is shifted away from the lateral patellofemoral joint in a similar fashion to the unloader, also leading to improvements in patellar tracking and enhanced proprioception. The authors asked patients to wear the reactive knee brace and after a mean follow up of 55 days found significant improvements in pain, participation in sports/activities, quality of life and an improvement in the Kujala score.

\section{Insoles}

In a similar fashion to knee braces, insoles are designed to shift force away from the damaged compartment of the knee and reduce joint stress. Laterally wedged insoles are currently used as an adjunct for the treatment of medial compartment knee OA by reducing knee adduction and the subsequent load on the medial side of the knee [41]. Hsu et al. also add that long term use of laterally wedged insoles leads to a permanent change in gait, with reduced knee adduction even without wearing insoles [42]. Several studies have also demonstrated efficacy in reducing pain experienced by knee OA patients immediately and also over a longer duration of 8 weeks [43]. Therefore the use of insoles may be a useful adjunct to treating knee OA nonconservatively.

\section{Electrical therapy}

Currently there are two broad classes of electrical therapy that are routinely used for OA of the knee. Transcutaneous Electrical Nerve Stimulation (TENS) is a neuromodulation technique (based on the 'Gate Control Theory' [44] which aims to reduce pain and improve function and has been shown to be very promising novel treatment. Chen et al. performed a systematic review and meta-analysis and found that the majority of studies demonstrated a significant reduction in knee OA pain following TENS usage [45]. One study in their analysis by Vance et al. found a reduction in pain in both TENS and placebo, but no significant difference between the groups [46]. However from their meta-analysis Chen et al. concluded there was no significant improvement in knee function or performance in the get up and go tests [45]. They found that while TENS appears to be effective at reducing pain, other methodologies are required as adjuncts to improve the functional capabilities of the knee. In contrast other individual RCTs were able to show significant improvements in knee function and exercise involvement when comparing TENS to placebo [47]. The same group also demonstrated that patients receiving TENS continued to use the device after the cessation of the trial period due to the symptomatic relief and that these patients also reduced the number and frequency of analgesic medications they were taking. Palmer et al. found that while TENS and sham-TENS combined with knee exercise and education led to improvements in pain and function, there were no significant difference in the control group of just knee exercise and education [48]. TENS may be a useful adjunct to treat knee pain in OA however there is uncertainty in the literature regarding the exact benefits and the work by Palmer et al. indicates that knee exercise and education should remain a staple of treatment [48]. It would be interesting if this group had also used a group solely with TENS and no additional treatment to compare the effects and this could potentially be investigated in the future.

Neuromuscular electrical stimulation (NMES) is slightly different from TENS and has the goal of eliciting muscle contractions to strengthen the quadriceps and hamstring muscles. Knee $\mathrm{OA}$ is associated with atrophy of the thigh muscle groups and pain and stiffness are often limiting factors in conventional exercise therapy. Following a period of NMES usage, Vaz et al. demonstrated that this treatment led to increased vastus lateralis muscle thickness, fascicle length, maximal knee extensor torque and was associated with a subsequent reduction in knee pain, stiffness and improvements in functional status [49]. Laufer et al. demonstrated that after finishing a 12 week programme of NMES, the results are partially maintained for a further 12 week period with respect to pain reduction and functional status; however the muscle strength, up-and-go test results and stair 
climbing ability were diminished [50]. This indicates that NMES is required to be used continually in patients with knee OA to maintain the beneficial effects of the treatment. Several other studies have demonstrated promises with this type of electrical therapy in the treatment of knee pain $[51,52]$.

While TENS and NMES are the most frequently used electrical modalities for treating knee OA pain, Zeng et al. concluded that another form of therapy, interferential current (IFC), was the most effective at relieving pain [53]. IFC delivers alternating mediumfrequency currents in a similar fashion to TENS to reduce pain. IFC can penetrate deeper into the tissues and appears to block peripheral nerves rather than the sensory and motor nerves that TENS acts on. However there is still very little useful data on the subject of IFC for treatment of knee OA and more research needs to be carried out before this modality is offered as a usual adjunct to the general public.

\section{Conclusions}

Chronic knee pain is a widespread complaint in the worldwide community and has been forecast to increase dramatically due to the ageing population and the obesity epidemic. Fortunately for the physician there are a wide variety of options available from the more conventional lifestyle modification and physiotherapy; to the newer methods such as electrical therapy and intra-articular injections. The key to effective treatment is to get the correct aetiology whether it be soft tissue-type or bony-type. Current management options demonstrated thus far have not been shown to be disease-modifying but are beneficial to the patient by improving knee symptoms and functional status. It is also crucial to delay TKR for as long as is acceptable to the patient in order to avoid the need for revision surgery and its associated complications, especially in the younger patient presenting with OA.

\section{References}

1. Jinks C, Jordan K, Ong BN, Croft P (2004) A brief screening tool for knee pain in primary care (KNEST). 2. Results from a survey in the general population aged 50 and over. Rheumatology (Oxford) 43: 55-61.

2. Porcheret M, Jordan K, Croft P; Primary Care Rhumatology Society (2007) Treatment of knee pain in older adults in primary care: development of an evidence-based model of care. Rheumatology (Oxford) 46: 638-648.

3. (2016) Arthritis Research. UK.

4. Peat G, Bergknut C, Frobell R, Jöud A, Englund M (2014) Populationwide incidence estimates for soft tissue knee injuries presenting to healthcare in southern Sweden: data from the Skåne Healthcare Register. Arthritis Res Ther 16: R162.

5. Silverwood V, Blagojevic-Bucknall M, Jinks C, Jordan JL, Protheroe J, et al. (2015) Current evidence on risk factors for knee osteoarthritis in older adults: a systematic review and meta-analysis. Osteoarthritis Cartilage 23: 507-515.

6. (2016) National Joint Registry.

7. Williams DP, Price AJ, Beard DJ, Hadfield SG, Arden NK, et al. (2013) The effects of age on patient-reported outcome measures in total knee replacements. Bone Joint J 95-95B: 38-44.

8. (2016) National Joint Registry. Patient characteristics for primary knee replacement procedures.

9. Khan M, Osman K, Green G, Haddad FS (2016) The epidemiology of failure in total knee arthroplasty: avoiding your next revision. Bone Joint $\mathrm{J}$ 98-98B: 105-12.

10. Beswick AD, Wylde V, Gooberman-Hill R, Blom A, Dieppe P (2012) What proportion of patients report long-term pain after total hip or knee replacement for osteoarthritis? A systematic review of prospective studies in unselected patients. BMJ Open 2: e000435.

11. Wylde V, Hewlett S, Learmonth ID, Dieppe P (2011) Persistent pain after joint replacement: prevalence, sensory qualities, and postoperative determinants. Pain 152: 566-572.

12. Husain A, Lee GC (2015) Establishing Realistic Patient Expectations Following Total Knee Arthroplasty. J Am Acad Orthop Surg 23: 707-713.

13. Messier SP, Gutekunst DJ, Davis C, DeVita P (2005) Weight loss reduces knee-joint loads in overweight and obese older adults with knee osteoarthritis. Arthritis Rheum 52: 2026-2032.

14. Aaboe J, Bliddal H, Messier SP, Alkjær T, Henriksen M (2011) Effects of an intensive weight loss program on knee joint loading in obese adults with knee osteoarthritis. Osteoarthritis Cartilage 19: 822-828.

15. DeVita P, Rider P, Hortobágyi T (2016) Reductions in knee joint forces with weight loss are attenuated by gait adaptations in class III obesity. Gait Posture 45: 25-30.

16. Atukorala I, Makovey J, Lawler L, Messier SP, Bennell K, et al. (2016) Is There a Dose-Response Relationship Between Weight Loss and Symptom Improvement in Persons With Knee Osteoarthritis? Arthritis Care Res (Hoboken) 68: 1106-1114.

17. Knoop J, Steultjens MP, Roorda LD, Lems WF, van der Esch M, et al. (2015) Improvement in upper leg muscle strength underlies beneficial effects of exercise therapy in knee osteoarthritis: secondary analysis from a randomised controlled trial. Physiotherapy 101: 171-177.

18. Smith TO, Postle K, Penny F, McNamara I, Mann CJ (2014) Is reconstruction the best management strategy for anterior cruciate ligament rupture? A systematic review and meta-analysis comparing anterior cruciate ligament reconstruction versus non-operative treatment. Knee 21: 4462-4470.

19. Page CJ, Hinman RS, Bennell KL (2011) Physiotherapy management of knee osteoarthritis. Int J Rheum Dis 14: 145-151.

20. Mutlu KE, Mustafaoglu R, Birinci T, Ozdincler AR (2016) Does Kinesio Taping of the Knee Improve Pain and Functionality in Patients with Knee Osteoarthritis?: A Randomized Controlled Clinical Trial. Am J Phys Med Rehabil.

21. Lee K, Yi CW, Lee S (2016) The effects of kinesiology taping therapy on degenerative knee arthritis patients' pain, function, and joint range of motion. J Phys Ther Sci 28: 63-66.

22. Cho HY, Kim EH, Kim J, Yoon YW (2015) Kinesio taping improves pain, range of motion, and proprioception in older patients with knee osteoarthritis: a randomized controlled trial. Am J Phys Med Rehabil 94: 192-200.

23. Kocyigit F, Turkmen MB, Acar M, Guldane N, Kose T, et al. (2015) Kinesio taping or sham taping in knee osteoarthritis? A randomized, double-blind, sham-controlled trial. Complement Ther Clin Pract 21: 262-267.

24. Wageck B, Nunes GS, Bohlen NB, Santos GM, de Noronha M (2016) Kinesio Taping does not improve the symptoms or function of older people with knee osteoarthritis: a randomised trial. J Physiother 62: 153-158.

25. Hassan BS, Mockett S, Doherty M (2002) Influence of elastic bandage on knee pain, proprioception, and postural sway in subjects with knee osteoarthritis. Ann Rheum Dis 61: 24-28.

26. Herrington L, Simmonds C, Hatcher J (2005) The effect of a neoprene sleeve on knee joint position sense. Res Sports Med 13: 37-46.

27. Pollo FE, Otis JC, Backus SI, Warren RF, Wickiewicz TL (2002) Reduction of medial compartment loads with valgus bracing of the osteoarthritic knee. Am J Sports Med 30: 414-421.

28. Pierrat B, Oullion R, Molimard J, Navarro L, Combreas M, et al. (2015) Characterisation of in-vivo mechanical action of knee braces regarding their anti-drawer effect. Knee 22: 80-87.

29. Hanzlíková I, Richards J, Tomsa M, Chohan A, May K, et al. (2016) The effect of proprioceptive knee bracing on knee stability during three different sport related movement tasks in healthy subjects and the 
Citation: Golding D, Lee PYF (2016) Non-surgical and Non-pharmacological Treatment of Knee Pain. J Arthritis 5: 225. doi: $10.4172 / 2167-7921.1000225$

Page 5 of 5

implications to the management of Anterior Cruciate Ligament (ACL) injuries. Gait Posture 48: 165-170.

30. Smith SD, Laprade RF, Jansson KS, Arøen A, Wijdicks CA (2014) Functional bracing of ACL injuries: current state and future directions. Knee Surg Sports Traumatol Arthrosc 22: 1131-1141.

31. Jansson KS, Costello KE, O'Brien L, Wijdicks CA, Laprade RF (2013) A historical perspective of PCL bracing. Knee Surg Sports Traumatol Arthrosc 21: 1064-1070.

32. Strobel MJ, Weiler A, Schulz MS, Russe K, Eichhorn HJ (2002) Fixed posterior subluxation in posterior cruciate ligament-deficient knees: diagnosis and treatment of a new clinical sign. Am J Sports Med 30: 32-38.

33. Jacobi M, Reischl N, Wahl P, Gautier E, Jakob RP (2010) Acute isolated injury of the posterior cruciate ligament treated by a dynamic anterior drawer brace: a preliminary report. J Bone Joint Surg Br 92: 1381-1384.

34. Petersen W, Ellermann A, Zantop T, Rembitzki IV, Semsch H, et al. (2016) Biomechanical effect of unloader braces for medial osteoarthritis of the knee: a systematic review (CRD 42015026136). Arch Orthop Trauma Surg 136: 649-656.

35. Raja K, Dewan N (2011) Efficacy of knee braces and foot orthoses in conservative management of knee osteoarthritis: a systematic review. Am J Phys Med Rehabil 90: 247-262.

36. Ramsey DK, Briem K, Axe MJ, Snyder-Mackler L (2007) A mechanical theory for the effectiveness of bracing for medial compartment osteoarthritis of the knee. J Bone Joint Surg Am 89: 2398-2407.

37. Steadman JR, Briggs KK, Pomeroy SM, Wijdicks CA (2016) Current state of unloading braces for knee osteoarthritis. Knee Surg Sports Traumatol Arthrosc 24: 42-50.

38. Squyer E, Stamper DL, Hamilton DT, Sabin JA, Leopold SS (2013) Unloader knee braces for osteoarthritis: do patients actually wear them? Clin Orthop Relat Res 471: 1982-1991.

39. Draganich L, Reider B, Rimington T, Piotrowski G, Mallik K, et al. (2006) The effectiveness of self-adjustable custom and off-the-shelf bracing in the treatment of varus gonarthrosis. J Bone Joint Surg Am 88: 2645-2652.

40. Khadavi MJ, Chen YT, Fredericson M (2015) A Novel Knee Orthosis in the Treatment of Patellofemoral Pain Syndrome. Open J Ther Rehab 3: 56-61.

41. Jones RK, Chapman GJ, Parkes MJ, Forsythe L, et al. (2015) The effect of different types of insoles or shoe modifications on medial loading of the knee in persons with medial knee osteoarthritis: a randomised trial. J Orthop Res 33: 1646-1654.
42. Hsu WC, Jhong YC, Chen HL, Lin YJ, Chen LF, et al. (2015) Immediate and long-term efficacy of laterally-wedged insoles on persons with bilateral medial kneeosteoarthritis during walking. Biomed Eng Online 14: 43 .

43. Cherian JJ, Jauregui JJ, Leichliter AK, Elmallah RK, Bhave A, et al. (2016) The effects of various physical non-operative modalities on the pain in osteoarthritis of the knee. Bone Joint J 98-98B: 89-94.

44. Melzack R, Wall PD (1965) Pain mechanisms: a new theory. Science 150: 971-979.

45. Chen LX, Zhou ZR, Li YL, Ning GZ, Li Y, et al. (2016) Transcutaneous Electrical Nerve Stimulation in Patients With Knee Osteoarthritis: Evidence From Randomized-controlled Trials. Clin J Pain 32: 146-154.

46. Vance CG, Rakel BA, Blodgett NP, DeSantana JM, Amendola A, et al. (2012) Effects of transcutaneous electrical nerve stimulation on pain, pain sensitivity, and function in people with knee osteoarthritis: a randomized controlled trial. Phys Ther 92: 898-910.

47. Cherian JJ, Harrison PE, Benjamin SA, Bhave A, Harwin SF, et al. (2016) Do the Effects of Transcutaneous Electrical Nerve Stimulation on Knee Osteoarthritis Pain and Function Last? J Knee Surg 29: 497-501.

48. Palmer S, Domaille M, Cramp F, Walsh N, Pollock J, et al. (2014) Transcutaneous electrical nerve stimulation as an adjunct to education and exercise for knee osteoarthritis: a randomized controlled trial. Arthritis Care Res (Hoboken) 66: 387-394

49. Vaz MA, Baroni BM, Geremia JM, Lanferdini FJ, Mayer A, et al. (2013) Neuromuscular electrical stimulation (NMES) reduces structural and functional losses of quadriceps muscle and improves health status in patients with knee osteoarthritis. J Orthop Res 31: 511-516.

50. Laufer Y, Shtraker H, Gabyzon EM (2014) The effects of exercise and neuromuscular electrical stimulation in subjects with knee osteoarthritis: a 3-month follow-up study. Clin Interv Aging 9: 1153-1161.

51. Imoto AM, Peccin MS, Teixeira LE, Silva KN, Abrahão M, et al. (2013) Is neuromuscular electrical stimulation effective for improving pain, function and activities of daily living ofknee osteoarthritis patients? A randomized clinical trial. Sao Paulo Med J 131: 80-87.

52. Bruce-Brand RA, Walls RJ, Ong JC, Emerson BS, O'Byrne JM, et al. (2012) Effects of home-based resistance training and neuromuscular electrical stimulation in knee osteoarthritis: a randomized controlled trial. BMC Musculoskelet Disord 13: 118.

53. Zeng C, Li H, Yang T, Deng ZH, Yang Y, et al. (2015) Electrical stimulation for pain relief in knee osteoarthritis: systematic review and network meta-analysis. Osteoarthritis Cartilage 23: 189-202. 